\title{
Locally constant $n$-operads as higher braided operads
}

\author{
Michael A. Batanin*
}

\begin{abstract}
We introduce a category of locally constant $n$-operads which can be considered as the category of higher braided operads. For $n=1,2, \infty$ the homotopy category of locally constant $n$-operads is equivalent to the homotopy category of classical nonsymmetric, braided and symmetric operads, respectively.
\end{abstract}

Mathematics Subject Classification (2010). 18D20, 18D50, 55P48.

Keywords. Braid groups, operads, homotopy category, recognition principle.

\section{Introduction}

It is well known that contractible nonsymmetric operads detect 1-fold loop spaces, contractible braided operads detect 2-fold loop spaces and that contractible symmetric operads detect $\infty$-fold loop spaces. A natural question arises: is there a sequence of groups $G^{(n)}=\left\{G_{k}^{(n)}\right\}_{k \geq 0}$ together with a notion of $G^{(n)}$-operad, which we would call $n$-braided operad, such that the algebras of a contractible such operad are $n$-fold loop spaces? With some natural minor assumptions one can prove that the answer to the above question is negative. This is because for such an operad $A$ the quotient $A_{k} / G_{k}^{(n)}$ is a $K\left(G_{k}^{(n)}, 1\right)$-space. One can show, however, that such a quotient must have a homotopy type of the space of unordered configurations of $k$ points in $\mathbb{R}^{n}$, which is a $K(\pi, 1)$-space only for $n=1,2, \infty$.

In this paper we show that there is a category of operads which we can think of as a correct replacement for the nonexistent category of $G^{(n)}$-operads in all dimensions. We call them locally constant $n$-operads. For $n=1,2, \infty$ the homotopy category of locally constant $n$-operads is equivalent to the homotopy category of classical nonsymmetric, braided and symmetric operads, respectively.

Here is a brief overview of the paper. In Section 2 we recall the definitions of symmetric and braided operads. In Section 3 we introduce the category of $n$-ordinals as higher dimensional analogue of the category of finite ordinals. Using this category and its subcategory of quasi-bijections we define $n$-operads and quasisymmetric $n$ operads in Section 4. In Section 5 we show that the category of quasi-bijections is

${ }^{*}$ The author holds the Scott Russell Johnson Fellowship in the Centre of Australian Category Theory at Macquarie University. 
closely related to the classical Fox-Neuwirth stratification of configuration spaces. As a corollary we observe that the nerve of this category has homotopy type of unordered configurations of points in $\mathbb{R}^{n}$. We also prove two technical lemmas that we use in Section 6 to relate different operadic notions. Finally in Section 7 we introduce locally constant operads and compare them with symmetric, braided and quasisymmetric operads. We also state our recognition principle for $n$-fold loop spaces.

\section{Symmetric and braided operads}

For a natural number $n$ we will denote by $[n]$ the ordinal

$$
0<1<\cdots<n .
$$

We denote an empty ordinal by $[-1]$. A morphism from $[n] \rightarrow[k]$ is any function between underlying sets. It can be order-preserving or not. It is clear that we then have a category. We denote this category by $\Omega^{s}$. Of course, $\Omega^{s}$ is equivalent to the category of finite sets. In particular, the symmetric group $S_{n+1}$ is the group of automorphisms of $[n]$.

Let $\sigma:[n] \rightarrow[k]$ be a morphism in $\Omega^{s}$ and let $0 \leq i \leq k$. Then the preimage $\sigma^{-1}(i)$ has a linear order induced from $[n]$. Hence, there exists a unique object $\left[n_{i}\right] \in \Omega^{s}$ and a unique order-preserving bijection $\left[n_{i}\right] \rightarrow \sigma^{-1}(i)$. We will call $\left[n_{i}\right]$ the fiber of $\sigma$ over $i$ and will denote it $\sigma^{-1}(i)$ or $\left[n_{i}\right]$.

Analogously, given a composite of morphisms in $\Omega^{s}$,

$$
[n] \stackrel{\sigma}{\longrightarrow}[l] \stackrel{\omega}{\longrightarrow}[k],
$$

we will denote $\sigma_{i}$ the $i$-th fiber of $\sigma$; i.e., the pullback

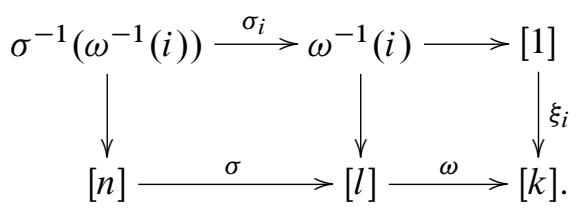

Let $S$ be the subcategory of bijections in $\Omega^{s}$. This is a strict monoidal groupoid with tensor product $\oplus$ given by ordinal sum and with $[-1]$ as its unital object.

A right symmetric collection in a symmetric monoidal category $V$ is a functor $A: \mathcal{S}^{\text {op }} \rightarrow V$. The value of $A$ on an object $[n]$ will be denoted $A_{n}$. Notice, that this is not a standard operadic notation. Classically, the notation for $A_{[n]}$ is $A_{n+1}$ to stress the fact that $A_{n+1}$ is the space of operations of arity $n+1$.

The following definition is classical May definition [7] of symmetric operad.

Definition 2.1. A (right) symmetric operad in $V$ is a right symmetric collection $A$ equipped with the following additional structure: 
- a morphism $e: I \rightarrow A_{0}$,

- for every order-preserving map $\sigma:[n] \rightarrow[k]$ in $\Omega^{s}$ a morphism

$$
\mu_{\sigma}: A_{k} \otimes\left(A_{n_{0}} \otimes \cdots \otimes A_{n_{k}}\right) \rightarrow A_{n},
$$

where $\left[n_{i}\right]=\sigma^{-1}(i)$.

They must satisfy the following identities:

(1) For any composite of order-preserving morphisms in $\Omega^{s}$

$$
[n] \stackrel{\sigma}{\longrightarrow}[l] \stackrel{\omega}{\longrightarrow}[k]
$$

the diagram

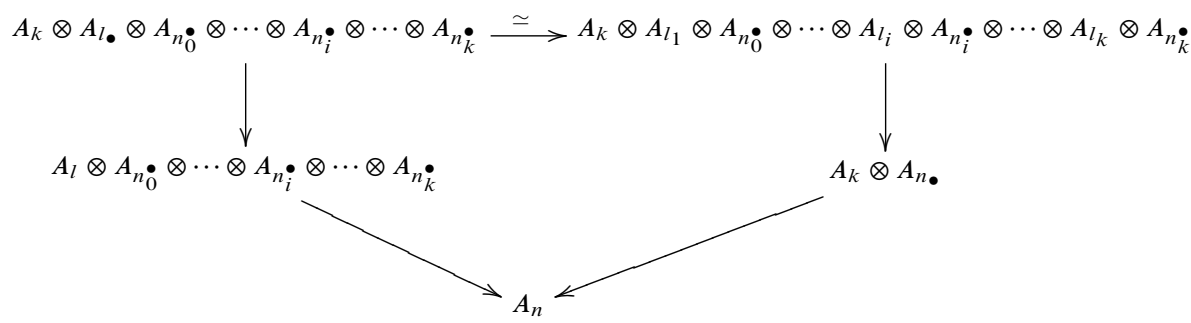

commutes. Here

$$
\begin{aligned}
A_{l_{\bullet}} & =A_{l_{0}} \otimes \cdots \otimes A_{l_{k}}, \\
A_{n_{i}^{\bullet}} & =A_{n_{i}^{0}} \otimes \cdots \otimes A_{n_{i}}^{m_{i}}
\end{aligned}
$$

and

$$
A_{n_{\bullet}}=A_{n_{0}} \otimes \cdots \otimes A_{n_{k}} .
$$

(2) For an identity $\sigma=\mathrm{id}:[n] \rightarrow[n]$ the diagram

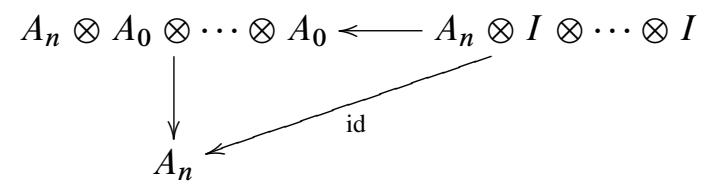

commutes.

(3) For the unique morphism $[n] \rightarrow[0]$ the diagram

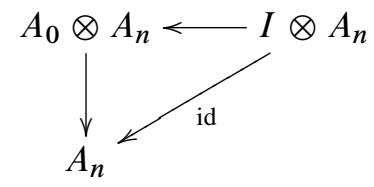

commutes. 
The following equivariance conditions are also required:

(1) For any order-preserving $\sigma:[n] \rightarrow[k]$ and any bijection $\rho:[k] \rightarrow[k]$ the following diagram commutes:

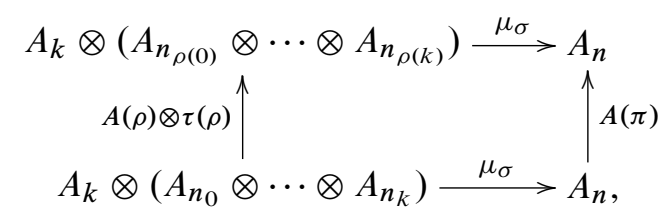

where $\tau(\rho)$ is the symmetry in $V$ which corresponds to permutation $\rho$ and $\pi=\Gamma_{S}(\rho ; 1, \ldots, 1)$ is the permutation, which permutes the fibers $\left[n_{0}\right], \ldots,\left[n_{k}\right]$ according to $\rho$ and whose restriction on each fiber is an identity.

(2) For any order-preserving $\sigma:[n] \rightarrow[k]$ and any set of bijections $\rho_{i}:\left[n_{i}\right] \rightarrow\left[n_{i}\right]$, $0 \leq i \leq k$, the following diagram commutes:

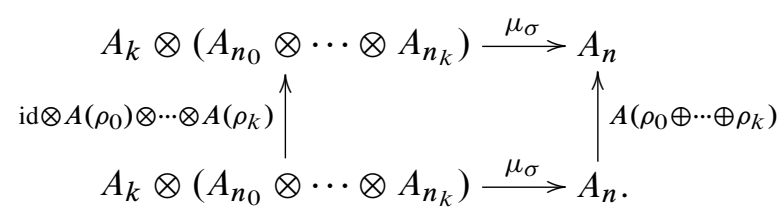

We can give an alternative definition of symmetric operad [2].

Definition 2.2. A (right) symmetric operad in $V$ is a right symmetric collection $A$ equipped with the following additional structure:

- a morphism $e: I \rightarrow A_{0}$,

- for every order-preserving map $\sigma:[n] \rightarrow[k]$ in $\Omega^{s}$ a morphism

$$
\mu_{\sigma}: A_{k} \otimes\left(A_{n_{0}} \otimes \cdots \otimes A_{n_{k}}\right) \rightarrow A_{n},
$$

where $\left[n_{i}\right]=\sigma^{-1}(i)$.

They must satisfy the same conditions as in the definition 2.1 with respect to order-preserving maps and identities but the equivariance conditions are replaced by the following:

(1) For every commutative diagram in $\Omega^{s}$,

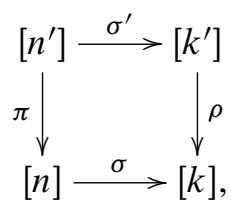


whose vertical maps are bijections and whose horizontal maps are order-preserving the following diagram commutes:

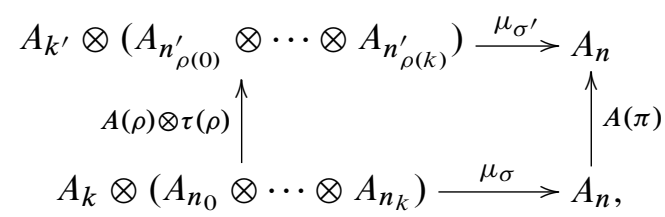

where $\tau(\rho)$ is the symmetry in $V$ which corresponds to permutation $\rho$.

(2) For every commutative diagram in $\Omega^{s}$,

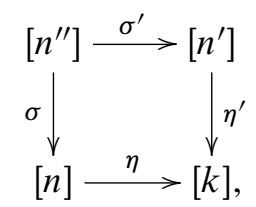

where $\sigma, \sigma^{\prime}$ are bijections and $\eta, \eta^{\prime}$ are order-preserving maps, the following diagram commutes:

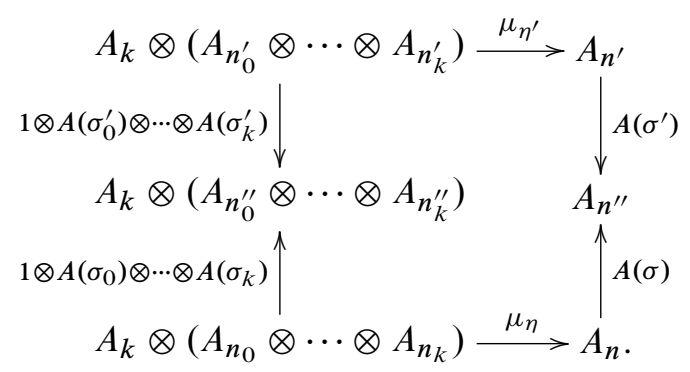

Proposition 2.1. Definitions 2.1 and 2.2 are equivalent.

We leave this proposition as an exercise for the reader.

Let $\mathcal{B r}$ be the groupoid of braid groups. We will regard the objects of $\mathscr{B r}$ as ordinals. There is a monoidal structure on $\mathscr{B r}$ given by ordinal sum on objects and concatenation of braids on morphism. The ordinal $[-1]$ is the unital object.

The following is the definition of braided operad from [4]. A right braided col-

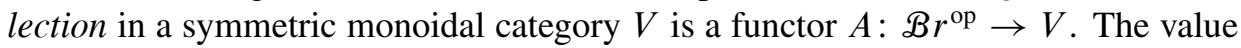
of $A$ on an object $[n]$ will be denoted $A_{n}$.

Definition 2.3. A right braided operad in $V$ is a right braided collection $A$ equipped with the following additional structure:

- a morphism $e: I \rightarrow A_{0}$, 
- for every order-preserving map $\sigma:[n] \rightarrow[k]$ in $\Omega^{s}$ a morphism

$$
\mu_{\sigma}: A_{k} \otimes\left(A_{n_{0}} \otimes \cdots \otimes A_{n_{k}}\right) \rightarrow A_{n},
$$

where $\left[n_{i}\right]=\sigma^{-1}(i)$.

They must satisfy the identities (1)-(3) from the definition 2.1 and the following two equivariancy conditions:

(1) For any order-preserving $\sigma:[n] \rightarrow[k]$ and any braid $\rho:[k] \rightarrow[k]$ the following diagram commutes:

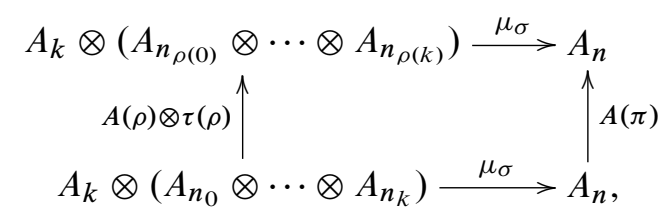

where $\tau(\rho)$ is the symmetry in $V$ which corresponds to the braid $\rho$ and $\pi=$ $\Gamma_{B}(\rho ; 1, \ldots, 1)$ is a braid obtained from $\rho$ by replacing the $i$-th strand of $\rho$ by $n_{i}$ parallel strands for each $i$.

(2) For any order-preserving $\sigma:[n] \rightarrow[k]$ and any set of braids $\rho_{i}:\left[n_{i}\right] \rightarrow\left[n_{i}\right]$, $0 \leq i \leq k$, the following diagram commutes:

$$
\begin{gathered}
A_{k} \otimes\left(A_{n_{0}} \otimes \cdots \otimes A_{n_{k}}\right) \stackrel{\mu_{\sigma}}{\longrightarrow} A_{n} \\
\stackrel{\uparrow}{\mathrm{id} \otimes A\left(\rho_{0}\right) \otimes \cdots \otimes A\left(\rho_{k}\right)} \mid \\
A_{k} \otimes\left(A_{\left.n_{0} \oplus \cdots \oplus \rho_{k}\right)} \otimes \cdots \otimes A_{n_{k}}\right) \stackrel{\mu_{\sigma}}{\longrightarrow} A_{n} .
\end{gathered}
$$

\section{3. n-ordinals and quasibijections}

Definition 3.1. An $n$-ordinal consists of a finite set $T$ equipped with $n$ binary relations $<_{0}, \ldots,<_{n-1}$ satisfying the following axioms:

(1) $<_{p}$ is nonreflexive;

(2) for every pair $a, b$ of distinct elements of $T$ there exists exactly one $p$ such that

$$
a<_{p} b \text { or } b<_{p} a \text {; }
$$

(3) if $a<_{p} b$ and $b<_{q} c$ then $a<_{\min (p, q)} c$.

Every $n$-ordinal can be represented as a pruned planar tree with $n$ levels. For example, the 2-ordinal

$$
0<_{0} 1, \quad 0<_{0} 2, \quad 0<_{0} 3, \quad 1<_{1} 2, \quad 2<_{1} 3, \quad 2<_{1} 3
$$


is represented by the pruned tree

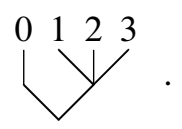

See [1] for a more detailed discussion.

Definition 3.2. A map of $n$-ordinals

$$
\sigma: T \rightarrow S
$$

is a map $\sigma: T \rightarrow S$ of underlying sets such that

$$
i<_{p} j \quad \text { in } T
$$

implies that

(1) $\sigma(i)<_{r} \sigma(j)$ for some $r \geq p$, or

(2) $\sigma(i)=\sigma(j)$, or

(3) $\sigma(j)<_{r} \sigma(i)$ for $r>p$.

For every $i \in S$ the preimage $\sigma^{-1}(i)$ (the fiber of $\sigma$ over $i$ ) has a natural structure of an $n$-ordinal.

We denote by $\operatorname{Ord}(n)$ the skeletal category of $n$-ordinals . The category $\operatorname{Ord}(n)$ is monoidal. The monoidal structure $\oplus$ is defined as follows. For two $n$-ordinals $S$ and $T$ the $n$-ordinal $S \oplus T$ has as an underlying set the union of underlying sets of $S$ and $T$. The orders $<_{k}$ restricted to the elements of $S$ and $T$ coincide with respective orders on $S$ and $T$, and $a<_{0} b$ if $a \in S$ and $b \in T$. The unital object for this monoidal structure is empty $n$-ordinal.

An $n$-ordinal structure on $T$ determines a linear order (called total order) on the elements of $T$ as follows:

$$
a<b \quad \text { iff } \quad a<_{r} b \quad \text { for some } 0 \leq r \leq n-1 .
$$

We denote by $[T]$ the set $T$ with its total linear order. In this way we have a monoidal functor

$$
[-]: \operatorname{Ord}(n) \rightarrow \Omega^{s} .
$$

This functor is faithful but not full. For example, no morphism from the 2-ordinal (2) to the 2-ordinal $0<_{1} 1$ can reverse the order of 1,2 and 3

We also introduce the category of $\infty$-ordinals $\operatorname{Ord}(\infty)$.

Definition 3.3. An $\infty$-ordinal consists of a finite set $T$ equipped with a sequence of binary relations $<_{0},<_{-1},<_{-2}, \ldots$ satisfying the following axioms:

(1) $<_{p}$ is nonreflexive; 
(2) for every pair $a, b$ of distinct elements of $T$ there exists exactly one $p$ such that

$$
a<_{p} b \text { or } b<_{p} a
$$

(3) if $a<_{p} b$ and $b<_{q} c$ then $a<_{\min (p, q)} c$.

The definition of morphism between $\infty$-ordinals coincides with the Definition 3.2. The category $\operatorname{Ord}(\infty)$ is the skeletal category of $\infty$-ordinals. As for $\operatorname{Ord}(n)$ we have a functor of total order

$$
[-]: \operatorname{Ord}(\infty) \rightarrow \Omega^{s}
$$

For a $k$-ordinal $R, k \leq n$ we consider its $(n-k)$-th vertical suspension $S^{n-k} R$, which is an $n$-ordinal with the underlying set $R$, and the order $<_{m}$ is equal to the order $<_{m-k}$ on $R$ (so $<_{m}$ is empty for $0 \leq m<n-k$ ). We also can consider the horizontal ( $n-1)$-suspension $T^{n-k} R$, which is a $n$-ordinal with the underlying set $R$, and the order $<_{m}$ is equal to the order on $R$ (so $<_{m}$ is empty for $k-1<m \leq n-1$ ).

The vertical suspension provides us with a functor $S: \operatorname{Ord}(n) \rightarrow \operatorname{Ord}(n+1)$. We also define an $\infty$-suspension functor $\operatorname{Ord}(n) \rightarrow \operatorname{Ord}(\infty)$ as follows. For an $n$-ordinal $T$ its $\infty$-suspension is an $\infty$-ordinal $S^{\infty} T$ whose underlying set is the same as the underlying set of $T$, and $a<_{p} b$ in $S^{\infty} T$ if $a<_{n+p-1} b$ in $T$. It is not hard to see that the sequence

$$
\operatorname{Ord}(0) \stackrel{S}{\longrightarrow} \operatorname{Ord}(1) \stackrel{S}{\longrightarrow} \operatorname{Ord}(2) \stackrel{S}{\longrightarrow} \cdots \stackrel{S}{\longrightarrow} \operatorname{Ord}(n) \longrightarrow \cdots \stackrel{S^{\infty}}{\longrightarrow} \operatorname{Ord}(\infty)
$$

exhibits $\operatorname{Ord}(\infty)$ as a colimit of $\operatorname{Ord}(n)$

Definition 3.4. A map of $n$-ordinals is called a quasibijection if it is a bijection of the underlying sets.

Let $Q_{n}, 1 \leq n \leq \infty$, be the subcategory of quasibijections of $\operatorname{Ord}(n)$. The total order functor induces then a functor which we will denote by the same symbol:

$$
[-]: Q_{n} \rightarrow S
$$

Definition 3.5. A map $\sigma$ of $n$-ordinals $1 \leq n \leq \infty$ is called order-preserving if it preserves the total orders in the usual sense, or, equivalently, only conditions 1 and 2 from the Definition 3.2 hold for $\sigma$.

Lemma 3.1. For every morphism $\sigma: T \rightarrow S$ in $\operatorname{Ord}(n), 1 \leq n \leq \infty$, there exists a factorisation

$$
T \stackrel{\pi}{\rightarrow} T^{\prime} \stackrel{v}{\rightarrow} S
$$

where $\pi$ is a quasibijection, $v$ is order-preserving and $\pi$ preserves total order on fibers of $v$. 
Proof. For $n=1$ this factorisation is trivial, since all maps of 1-ordinals are orderpreserving.

Let $n=2$. Let $\sigma: T \rightarrow S$ be a map of 2-ordinals and let $S=S[k]$ be a suspension of the 1-ordinal $[k]$. Let $T^{\prime}$ be the 2-ordinal whose underlying set is the same as that of $T$, whose only nonempty order is $<_{1}$ and whose total order coincides with $[T]$. So $T^{\prime}$ itself is a vertically suspended 1 -ordinal. Now one can factorise the $\operatorname{map}[\sigma]:[T] \rightarrow[S]$ in $\Omega^{s}$ :

$$
[T] \stackrel{\pi}{\rightarrow}\left[T^{\prime}\right] \stackrel{\nu}{\rightarrow}[S],
$$

with $v$ being total order-preserving and $\pi$ a bijection which preserves the order on the fibers of $\sigma$ [2]. Obviously, $v$ can be considered as a map of 2-ordinals and it is order-preserving. Let us check that $\pi$ is also a map of 2-ordinals. Indeed, if $i, j$ are from the same fiber of $\sigma$ then $\pi$ preserves their order. If $i<_{0} j$ in $T$ and they are from different fibers, then there is no restriction on $\pi$ since $T^{\prime}$ is a suspended 1-ordinal. Finally, if $i<_{1} j$ in $T$ and they are from different fibers then $\sigma(i)<_{1} \sigma(j)$, so $\pi(i)<_{1} \pi(j)$ because $v$ is order-preserving.

Finally, if $S$ is an arbitrary 2-ordinal, then $S=S_{1} \oplus \cdots \oplus S_{k}$ for some suspended 1-ordinals $S_{1}, \ldots, S_{k}$ and moreover,

$$
\sigma=\sigma_{1} \oplus \cdots \oplus \sigma_{k}: T=T_{1} \oplus \cdots \oplus T_{k} \rightarrow S_{1} \oplus \cdots \oplus S_{k} .
$$

By applying the previous result to each $\sigma_{k}$ we obtain a required factorisation of $\sigma$.

The factorisation for $n>2$ can be obtained similarly.

\section{Quasisymmetric $n$-operads}

We now recall the definition of pruned $(n-1)$-terminal $n$-operad [1]. Since we do not need other types of $n$-operads in this paper we will call them simply $n$-operads. The notation $U_{n}$ means the terminal $n$-ordinal.

Let $V$ be a symmetric monoidal category. For a morphism of $n$-ordinals $\sigma: T \rightarrow$ $S$ the $n$-ordinal $T_{i}$ is the fiber $\sigma^{-1}(i)$.

Definition 4.1. An $n$-operad in $V$ is a collection $A_{T}, T \in \operatorname{Ord}(n)$, of objects of $V$ equipped with the following structure:

- a morphism $e: I \rightarrow A_{U_{n}}$ (the unit),

- for every morphism $\sigma: T \rightarrow S$ in $\operatorname{Ord}(n)$ a morphism

$$
m_{\sigma}: A_{S} \otimes A_{T_{0}} \otimes \cdots \otimes A_{T_{k}} \rightarrow A_{T} \quad \text { (the multiplication). }
$$

They must satisfy the following identities: 
- for any composite $T \stackrel{\sigma}{\rightarrow} S \stackrel{\omega}{\rightarrow} R$, the associativity diagram

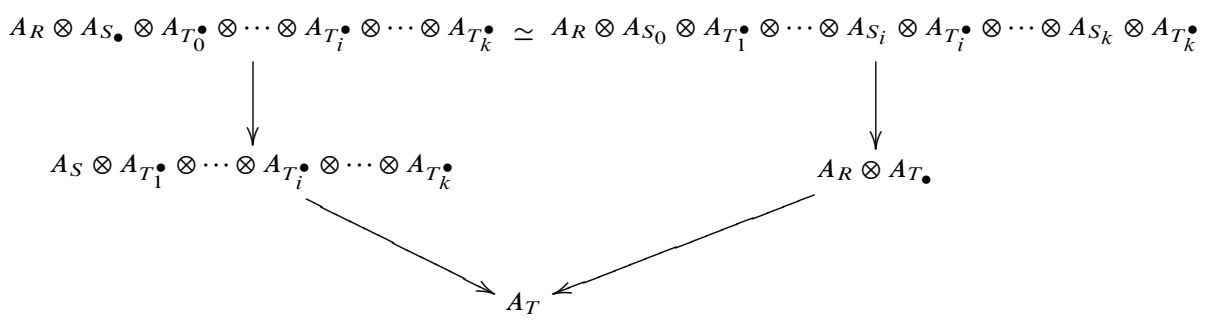

commutes, where

and

$$
\begin{aligned}
& A_{S_{\bullet}}=A_{S_{0}} \otimes \cdots \otimes A_{S_{k}}, \\
& A_{T_{i}^{*}}=A_{T_{i}^{0}} \otimes \cdots \otimes A_{T_{i}^{m_{i}}},
\end{aligned}
$$

$$
A_{T_{\bullet}}=A_{T_{0}} \otimes \cdots \otimes A_{T_{k}} ;
$$

- for an identity $\sigma=\mathrm{id}: T \rightarrow T$ the diagram

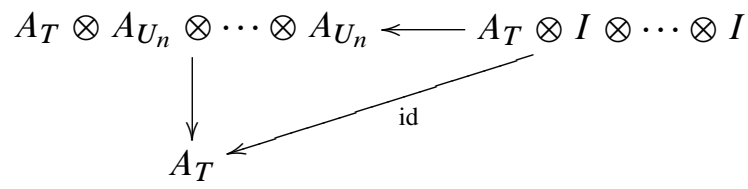

commutes;

- for the unique morphism $T \rightarrow U_{n}$ the diagram

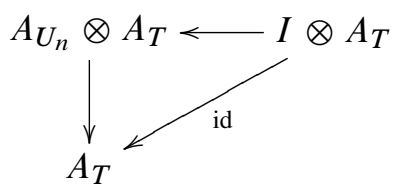

commutes.

Let $\sigma: T \rightarrow S$ be a quasibijection and $A$ be a pruned $n$-operad. Since a fiber of $\sigma$ is the terminal $n$-ordinal $U_{n}$, the multiplication

$$
\mu_{\sigma}: A_{S} \otimes\left(A_{U_{n}} \otimes \cdots \otimes A_{U_{n}}\right) \rightarrow A_{T}
$$

in composition with the morphism

$$
A_{S} \rightarrow A_{S} \otimes(I \otimes \cdots \otimes I) \rightarrow A_{S} \otimes\left(A_{U_{n}} \otimes \cdots \otimes A_{U_{n}}\right)
$$

induces a morphism

$$
A(\sigma): A_{S} \rightarrow A_{T} .
$$

It is not hard to see that in this way $A$ becomes a contravariant functor on $Q_{n}$. 
Definition 4.2. We call a pruned $n$-operad $A$ quasisymmetric if for every quasibijection $\sigma: T \rightarrow S$ the morphism

$$
A(\sigma): A_{S} \rightarrow A_{T}
$$

is an isomorphism.

The desymmetrisation functor from symmetric to $n$-operads for finite $n$ was defined in [2] using pulling back along the functor [-] $: \operatorname{Ord}(n) \rightarrow \Omega^{s}$. It was shown that this functor has a left adjoint which we call symmetrisation. We can obviously extend these definitions to $n=\infty$. By construction the desymmetrisation of a symmetric operad is a quasisymmetric $n$-operad for any $n$.

Let $\Pi Q_{n}$ be the fundamental groupoid of $Q_{n}$. A quasisymmetric operad provides, therefore, a contravariant functor on $\Pi Q_{n}$.

Definition 4.3. A $Q_{n}$-collection is a contravariant functor on $Q_{n}$. А $\Pi Q_{n}$-collection is a contravariant functor on $\Pi Q_{n}$.

Definition 4.4. A $Q_{n}$-operad is a $\Pi Q_{n}$-collection $A$ together with the following structure:

- for every order-preserving map $\sigma: T \rightarrow S$ the usual operadic map

$$
\mu_{\sigma}: A_{S} \otimes\left(A_{T_{0}} \otimes \cdots \otimes A_{T_{k}}\right) \rightarrow A_{T} .
$$

This collection of maps must satisfy the usual associativity and unitarity conditions plus two equivariancy conditions:

- For every commutative diagram

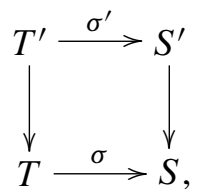

where vertical maps are quasi-bijections and horizontal maps are order-preserving, the diagram

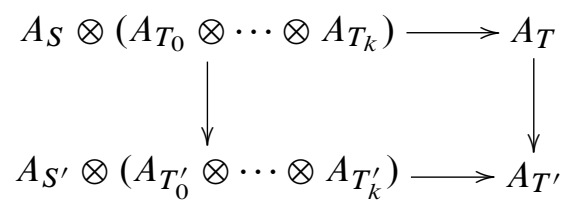

commutes. 
- For every commutative diagram

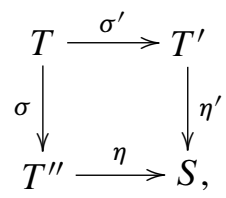

where $\sigma, \sigma^{\prime}$ are quasi-bijections and $\eta, \eta^{\prime}$ are order-preserving, the diagram

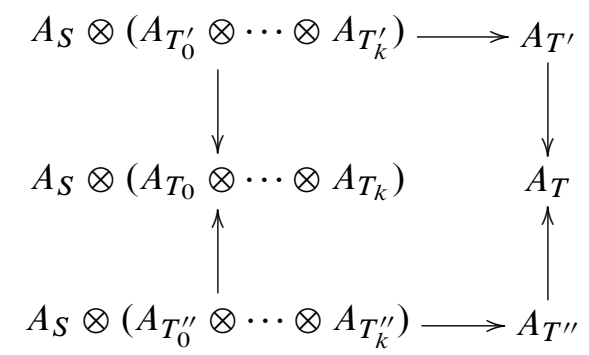

commutes.

Theorem 4.1. The category of $Q_{n}$-operads is equivalent to the category of quasisymmetric n-operads.

Proof. Obviously, every quasisymmetric $n$-operad is a $Q_{n}$-operad. Let us construct an inverse functor. Given a $Q_{n}$-operad $C$ we define a quasisymmetric operad $A$ on an $n$-ordinal $T$ to be equal to $C_{T}$. We have to define $A$ on an arbitrary map of $n$-ordinals $\sigma: T \rightarrow S$.

Let us choose a factorisation of $\sigma$ according to Lemma 3.1.

Now we can define operadic multiplication by the following commutative diagram:

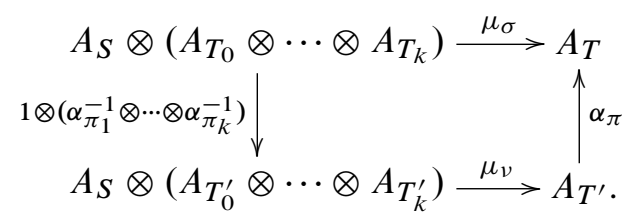

The second equivariancy axiom implies that this definition does not depend on a chosen factorisation. Suppose now that we have a composite

$$
T \stackrel{\sigma}{\rightarrow} S \stackrel{\omega}{\rightarrow} R .
$$


It generates the following factorization diagram

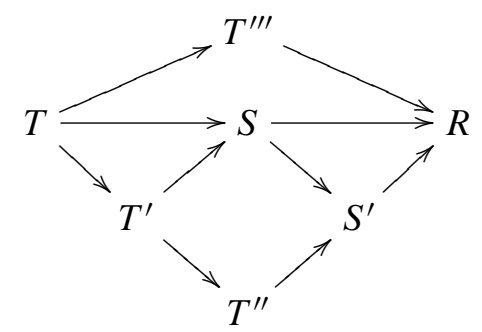

which in its turn generates the following huge diagram:

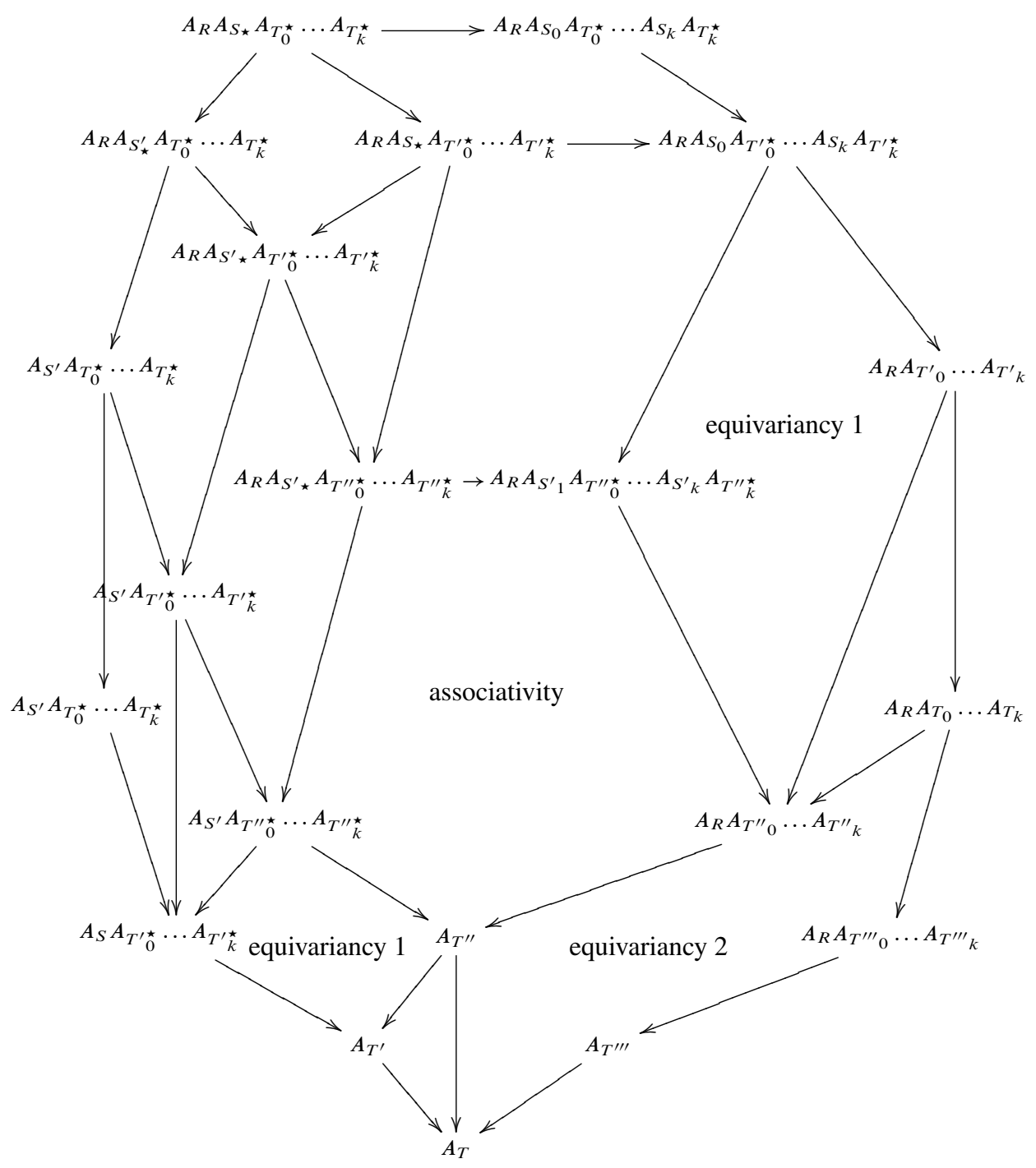


In this diagram we omit the symbol $\otimes$ to shorten the notations. Then the central region of the diagram commutes because of associativity of $A$ with respect to orderpreserving maps of $n$-ordinals. Other regions commute either by one of equivariancy conditions either by naturality either by functoriality. The commutativity of this diagram means the associativity of $A$ with respect to composition of maps of $n$ ordinals.

\section{The category of quasi-bijections and configuration spaces}

It is clear that the category $Q_{n}$ is the union of connected components $Q_{n}(k)$ where $k$ is the cardinality of the $n$-ordinals.

Theorem 5.1. (1) For a finite $n$ the space $N\left(Q_{n}(k)\right)$ has homotopy type of unordered configuration spaces of $k$-points in $\mathbb{R}^{n}$.

(2) The localisation functor

$$
l_{2}: Q_{2} \rightarrow \Pi Q_{2}
$$

induces a weak equivalence of the nerves.

(3) The groupoid $\Pi Q_{2}$ is equivalent to the groupoid of braids.

(4) The localisation functor

$$
l_{\infty}: Q_{\infty} \rightarrow \Pi Q_{\infty}
$$

induces a weak equivalence of the nerves.

(5) The groupoids $\Pi Q_{n}, 3 \leq n \leq \infty$, are equivalent to the symmetric groups groupoid.

Proof. We give a sketch of the proof. A detailed discussion can be found in [1], [3]. Consider the configuration space of ordered $k$-points in $\mathbb{R}^{n}$ :

$$
\operatorname{Conf}_{k}\left(\mathbb{R}^{n}\right)=\left\{\left(x_{1}, \ldots, x_{k}\right) \in\left(\mathbb{R}^{n}\right)^{k} \mid x_{i} \neq x_{j} \text { if } i \neq j\right\} .
$$

It admits a so-called Fox-Neuwirth stratification.

Let $S_{+}^{n-p-1}$ denote the open $(n-p-1)$-hemisphere in $\mathbb{R}^{n}, 0 \leq p \leq n-1$ :

$$
\stackrel{o}{S}_{+}^{n-p-1}=\left\{x \in \mathbb{R}^{n} \mid x_{1}^{2}+\cdots+x_{n}^{2}=1, x_{p+1}>0, x_{i}=0 \text { if } 1 \leq i \leq p\right\} .
$$

Similarly,

$$
\stackrel{o}{S}_{-}^{n-p-1}=\left\{x \in \mathbb{R}^{n} \mid x_{1}^{2}+\cdots+x_{n}^{2}=1, x_{p+1}<0, x_{i}=0 \text { if } 1 \leq i \leq p\right\} .
$$

Let $u_{i j}: \operatorname{Conf}_{k}\left(\mathbb{R}^{n}\right) \rightarrow S^{n-1}$ be the function

$$
u_{i j}\left(x_{1}, \ldots, x_{k}\right)=\frac{x_{j}-x_{i}}{\left\|x_{j}-x_{i}\right\|} .
$$


The Fox-Neuwirth cell corresponding to an $n$-ordinal $T$ with $[T]=[k-1]$ is a subspace of $\operatorname{Conf}_{k}\left(\mathbb{R}^{n}\right)$,

$$
\begin{array}{r}
\mathrm{FN}_{T}=\left\{x \in \operatorname{Conf}_{k}\left(\mathbb{R}^{n}\right) \mid u_{i j}(x) \in \stackrel{o}{S}_{+}^{n-p-1} \text { if } i<_{p} j \text { in } T,\right. \\
\left.u_{i j}(x) \in \stackrel{o}{S}_{-}^{n-p-1} \text { if } j<_{p} i \text { in } T\right\} .
\end{array}
$$

Each Fox-Neuwirth cell is an open convex subspace of $\left(\mathbb{R}^{n}\right)^{k}$. We also have

$$
\operatorname{Conf}_{k}\left(\mathbb{R}^{n}\right)=\bigcup_{[T]=[k-1], \pi \in S_{k}} \pi \mathrm{FN}_{T} .
$$

Here $\pi \mathrm{FN}_{T}$ means a space obtained from $\mathrm{FN}_{T}$ by renumbering points according to the permutation $\pi$.

Let $J_{n}(k)$ be the Milgram poset of all possible $n$-ordinal structures on the set $\{0, \ldots, k-1\}$ [1]. The group $S_{k}$ acts on $J_{n}(k)$ and the quotient $J_{n}(k) / S_{k}$ is isomorphic to $Q_{n}(k)$.

One can think of an element from $J_{n}(k)$ as a pair $(T, \pi)$ where $T$ is an $n$-ordinal and $\pi$ is a permutation from $S_{k}$ and $(T, \pi)>(S, \xi)$ in $J_{n}(k)$ when there exists a quasibijection $\sigma: T \rightarrow S$ and $\xi \cdot \pi=\sigma$.

We also can associate a convex subspace of the configuration space $\mathrm{FN}(T, \pi)=$ $\pi \mathrm{FN}_{T}$ with every element of $J_{n}(k)$. Moreover, if $(T, \pi)>(S, \xi)$ then $\mathrm{FN}(S, \xi)$ is on the boundary of the closure of $\operatorname{FN}(T, \pi)$. Let us define

$$
\overline{\mathrm{FN}}(T, \pi)=\bigcup_{(S, \xi) \leq(T, \pi)} \mathrm{FN}(S, \xi) .
$$

The spaces $\overline{\mathrm{FN}}(T, \pi)$ are contractible and, moreover, we have a functor

$$
\overline{\mathrm{FN}}: J_{n}^{\mathrm{op}}(k) \rightarrow \text { Top. }
$$

We then have the following zig-zag of weak equivalences

$$
N\left(J_{n}^{\mathrm{op}}(k)\right) \leftarrow \operatorname{hocolim} \overline{\mathrm{FN}} \rightarrow \operatorname{colim} \overline{\mathrm{FN}} \simeq \operatorname{Conf}_{k}\left(\mathbb{R}^{n}\right) .
$$

The first statement of the theorem follows then from the quotient of the zig-zag above by the action of the symmetric group. The second and the third statements are the consequences of the fact that the space $\operatorname{Conf}_{k}\left(\mathbb{R}^{2}\right)$ is the $K\left(\mathscr{B} r_{k}, 1\right)$-space. The fifth statement follows from the fact that the fundamental group of $\operatorname{Conf}_{k}\left(\mathbb{R}^{n}\right)$ is trivial for $n>3$. Finally the fourth statement can be obtained using the formula $Q_{\infty}=\operatorname{colim}_{n} Q_{n}$.

We shall now, in Lemmas 5.1 and 5.2, make the equivalence between $\Pi Q_{2}$ and $\mathscr{B r}$ more explicit. These results will then be used in Section 6 to relate different operadic notions.

The total order functor [-]: $Q_{2} \rightarrow \S$ induces by the universal property a functor $s_{2}: \Pi Q_{2} \rightarrow \delta$. 


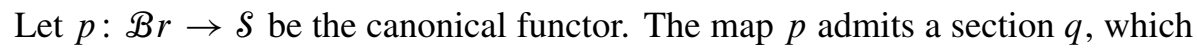
is not a homomorphism. For $\sigma \in S_{n}$ we construct a braid $q(\sigma)$ which, for $i<j$ such that $\sigma(i)>\sigma(j)$, has a strand from $i$ to $\sigma(i)$ which goes over the strand from $j$ to $\sigma(j)$ and there is no crossing if $\sigma$ preserves the order of $i$ and $j$.

Lemma 5.1. The composite

$$
Q_{2} \stackrel{[-]}{\longrightarrow} S \longrightarrow q \mathscr{B} r
$$

is a functor.

The functor induced by the universal property of $\Pi Q_{2}$,

$$
b: \Pi Q_{2} \rightarrow \mathscr{B} r,
$$

is an equivalence of groupoids.

Proof. To prove that $q[-]$ is a functor we have to prove that it preserves composition. We observe that in a composite of quasi-bijections of 2-ordinals $T \stackrel{\sigma}{\rightarrow} S \stackrel{\xi}{\rightarrow} R$ if $\sigma$ reverses the total order of two elements $i, j \in T$ then $\xi$ cannot reverse the order of $\sigma(i)$ and $\sigma(j)$. So, the resulting overcrossings in the composite $q[\sigma] q[\xi]$ are the same as in $q[\sigma \cdot \xi]$.

To prove the second claim it is sufficient to check that the induced morphism of groups

$$
b: \Pi Q_{2}(S[n-1], S[n-1]) \rightarrow \mathscr{B} r_{n}
$$

is an isomorphism.

It is obviously an epimorphism. So we have to prove that it is also a monomorphism.

For this it will be enough to prove that if a zig-zag

$$
z: S[n-1] \leftarrow T[n-1] \rightarrow S[n-1] \leftarrow \cdots \rightarrow S[n-1],
$$

where each arrow is given by a permutation of two consecutive elements or an identity permutation, is such that the corresponding braid $b(z)$ is trivial, then $z$ is trivial in $\Pi Q_{2}$.

This can be done if we prove that the morphisms in $\Pi Q_{2}(S[n-1], S[n-1])$,

$$
\bar{\sigma}_{i}: S[n-1] \stackrel{1}{\leftarrow} T[n-1] \stackrel{\sigma_{i}}{\rightarrow} S[n-1],
$$

where the left arrow is given by an identity and the right arrow is given by permutation $\sigma_{i}$ which changes the order of $i$ and $i+1$, satisfy the classical Artin braid relations. Then we can prove triviality of $z$ using the same rewriting process as for $b(z)$. 
Let $j>i+1$ and choose $m, l$ such that $[m-1] \oplus[l-1]=[n-1]$ and $i \in[m-1]$, $j=m+1$. The following commutative diagram in $Q_{2}$ proves that $\bar{\sigma}_{i} \bar{\sigma}_{j}=\bar{\sigma}_{j} \bar{\sigma}_{i}$ :

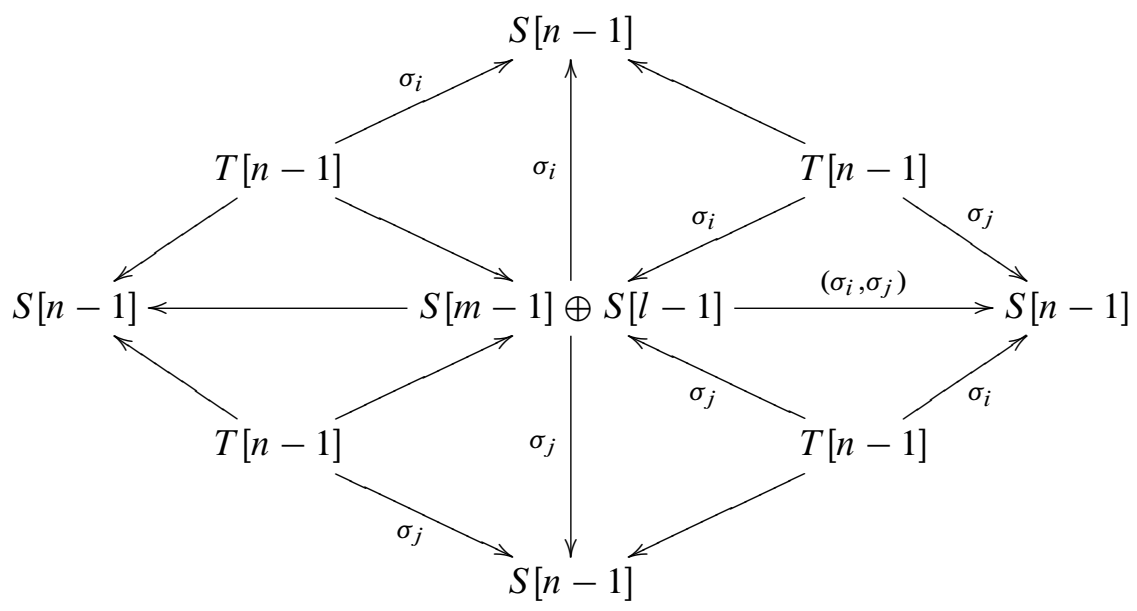

In this diagram all unnamed morphisms are identities on the underlying sets. The morphism $\left(\sigma_{i}, \sigma_{j}\right)$ acts as $\sigma_{i}$ on $[m-1]$ and as $\sigma_{0}$ on $[l-1]$.

For the proof of the Yang-Baxter relations $\bar{\sigma}_{i} \bar{\sigma}_{i+1} \bar{\sigma}_{i}=\bar{\sigma}_{i+1} \bar{\sigma}_{i} \bar{\sigma}_{i+1}$ we should consider the following commutative diagram in $Q_{2}$, which expresses the morphism $\bar{\sigma}_{i+1} \bar{\sigma}_{i} \bar{\sigma}_{i+1}$ :

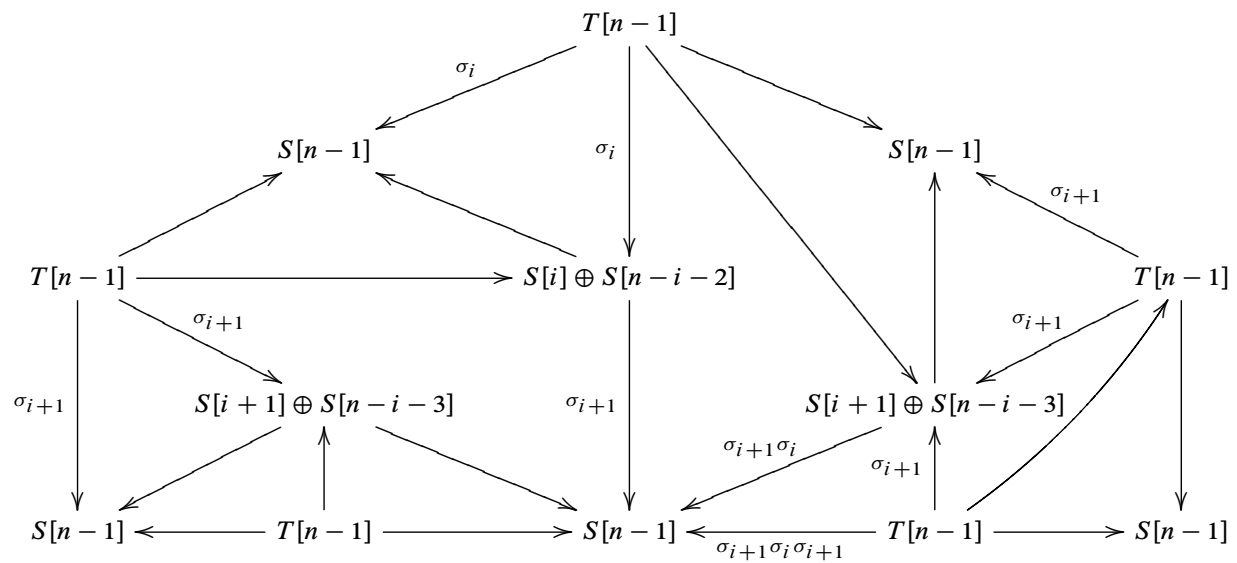

An analogous diagram (the mirror image of the above diagram) can be written for $\bar{\sigma}_{i} \bar{\sigma}_{i+1} \bar{\sigma}_{i}$. The relation follows from it immediately. 
So we have a commutative diagram of categories and functors

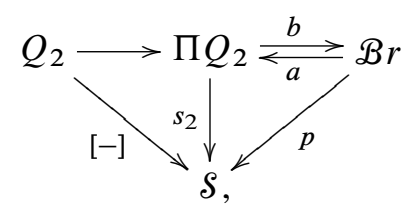

where $c$ is an adjoint equivalence to $b$. Notice that all functors in this diagram are strict monoidal functors.

Lemma 5.2. Let

$$
z: S \stackrel{\sigma}{\leftarrow} T \stackrel{\eta}{\rightarrow} R
$$

be a zig-zag of quasi-bijections of n-ordinals such that

$$
s_{2}(z)=\tau_{1} \oplus \cdots \oplus \tau_{k} .
$$

Then there exist braids $b_{i}, 1 \leq i \leq k$, such that $p\left(b_{i}\right)=\tau_{i}, 1 \leq i \leq k$, and

$$
b(z)=b_{1} \oplus \cdots \oplus b_{k} .
$$

Proof. Our aim is to prove that there exist quasi-bijections $\sigma_{i}: T_{i} \rightarrow S_{i}=S\left[n_{i}\right]$, $\eta_{i}: T_{i} \rightarrow R_{i}=S\left[n_{i}\right], 1 \leq i \leq k, \xi: \bigoplus_{i} S_{i} \rightarrow S, \zeta: \bigoplus_{i} R_{i} \rightarrow R$, and $\kappa: \bigoplus_{i} T_{i} \rightarrow T$ such that the diagram

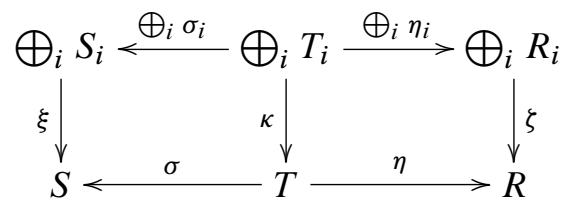

commutes and $b(\xi)=b(\zeta)=\Gamma_{B}(\pi ; 1, \ldots, 1)$ for a braid $\pi$ on $k$ strands. Then the result will follow from an elementary observation that the braid

$$
b(S) \stackrel{b(\xi)^{-1}}{\longrightarrow} \bigoplus_{i} b\left(S_{i}\right) \stackrel{\oplus_{i} b\left(\sigma_{i}\right)^{-1}}{\longrightarrow} \bigoplus_{i} b\left(T_{i}\right) \stackrel{\oplus_{i} b\left(\eta_{i}\right)}{\longrightarrow} \bigoplus_{i} b\left(R_{i}\right) \stackrel{b(\xi)}{\longrightarrow} b(R)
$$

is equal to

$$
\bigoplus_{i} b\left(S_{i}\right) \stackrel{\oplus_{i} b\left(\sigma_{i}^{-1}\right)}{\longrightarrow} \bigoplus_{i} b\left(T_{i}\right) \stackrel{\oplus_{i} b\left(\eta_{i}\right)}{\longrightarrow} \bigoplus_{i} b\left(R_{i}\right) .
$$

It is enough to proof the lemma for $k=2$. The rest follows by induction. Also without loss of generality we can assume that $S=S[n]$ and $T=T[n]$. Now $p(S)$ is the ordinal sum $[l] \oplus[m], n=m+1+1$, and the image of the restriction of the map $\sigma^{-1} \eta$ on $\{0, \ldots, l\}$ is $\{0, \ldots, l\}$, while the image of the restriction on $\{l+1, \ldots, m+l+1\}$ is $\{l+1, \ldots, m+l+1\}$. 
We put $S_{1}=S[l], T_{1}=T[l]$ and $S_{2}=S[m], T_{1}=T[m]$. We have to construct quasi-bijections

$$
\sigma_{i}, \eta_{i}: T_{i} \rightarrow S_{i}, \quad i=1,2,
$$

and also quasi-bijections

$$
\xi, \zeta: S_{1} \oplus S_{2} \rightarrow S, \quad \kappa: T_{1} \oplus T_{2} \rightarrow T,
$$

which make the diagram

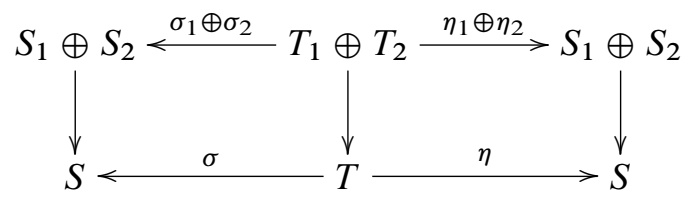

commutative.

The quasibijection $\kappa$ is simply the identity. Let us describe $\sigma_{1}$. Let $\sigma([l])$ be the image of the set $\{0, \ldots, l\}$ in the ordinal $[n]$. This image gets an induced order from $[n]$ which makes it isomorphic to $[l]$. Let $\phi_{1}: \sigma([l]) \rightarrow[l]$ be this unique isomorphism. We define $\sigma_{1}$ as the composite

$$
[l] \rightarrow \sigma([l]) \stackrel{\phi_{1}}{\rightarrow}[l] .
$$

Similarly, we define $\sigma_{2}$ as the composite

$$
[m] \rightarrow \sigma([m]) \stackrel{\phi_{2}}{\rightarrow}[m],
$$

where $\sigma([m])$ is the image of $\{l+1, \ldots, m+l+1\}$, and we give analogous definitions for $\eta_{1}$ and $\eta_{2}$.

Finally, we define $\xi$ by the formula

$$
\xi(x)= \begin{cases}\phi_{1}^{-1}(x) & \text { if } x \in\{0, \ldots, l\}, \\ \phi_{2}^{-1}(x) & \text { if } x \in\{l+1, \ldots, m+l+1\} .\end{cases}
$$

We use a similar argument to define $\zeta$. The commutativity of the diagram (3) follows from the definition.

\section{Quasisymmetric $n$-operads vs symmetric and braided operads}

Theorem 6.1. The category of quasisymmetric 2-operads and the category of braided operads are equivalent.

Proof. We first prove that the category of quasisymmetric 2-operads is equivalent to the category whose objects are mixed 2-operads in the sense of the definition below and whose morphisms are multiplications and units preserving morphisms of the underlying braided collections. 
Definition 6.1. A mixed 2-operad in $V$ is a right braided collection $A$ equipped with the following additional structure:

- a morphism $e: I \rightarrow A_{0}$,

- for every order-preserving map $\sigma:[n] \rightarrow[k]$ in $\Omega^{s}$ a morphism

$$
\mu_{\sigma}: A_{k} \otimes\left(A_{n_{0}} \otimes \cdots \otimes A_{n_{k}}\right) \rightarrow A_{n},
$$

where $\left[n_{i}\right]=\sigma^{-1}(i)$.

They must satisfy the identities (1-3) from the definition of symmetric operad and the following two equivariance conditions:

(1) For any two quasi-bijections of 2-ordinals $\pi, \rho$ and two order-preserving maps $\sigma, \sigma^{\prime} \in \Omega^{s}$ such that the diagram

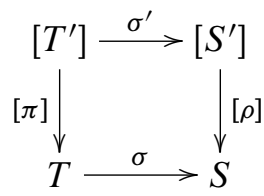

commutes in $\Omega^{s}$ the following induced diagram commutes:

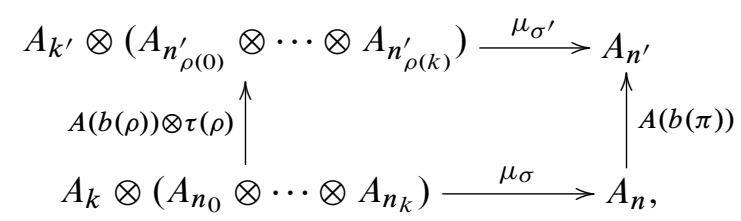

where $\tau(\rho)$ is the symmetry in $V$ which corresponds to the permutation $[\rho]$.

(2) For any two quasi-bijections $\sigma, \sigma^{\prime}$ and two order-preserving maps $\eta, \eta^{\prime} \in \Omega^{s}$ such that the diagram

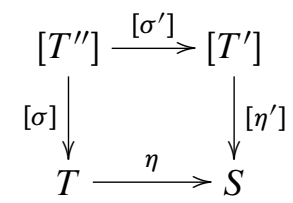

commutes in $\Omega^{s}$ the following diagram commutes:

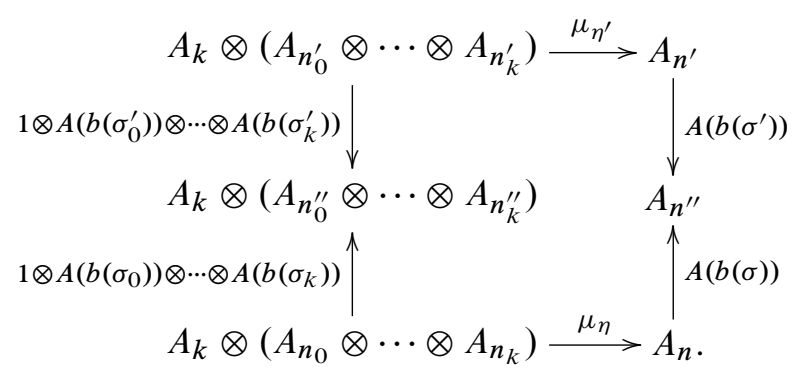


For a quasisymmetric 2-operad $A$ we define a mixed 2-operad $B$ by pulling back along the equivalence $c: B r \rightarrow \Pi Q_{2}$. And vice versa, we produce a quasisymmetric 2-operad from a mixed 2-operad by pulling back along $b: \Pi Q_{2} \rightarrow B r$. It is not hard to check that this indeed gives the necessary equivalence of the corresponding operadic categories. Now we will prove that the category of mixed 2-operads is equivalent to the category of braided operads. Let $A$ be an operad in the sense of 6.1. We have to check that $A$ also satisfies the Fiedorowicz equivariance conditions. Let us start from the second condition. For each $\rho_{i}$ let us choose a zigzag of $2 L$ morphisms in $Q_{2}$ such that

$$
\rho_{i}=b\left(T_{i} \stackrel{\tau_{1}}{\longleftarrow} R_{i}^{1} \stackrel{\tau_{2}}{\longrightarrow} R_{i}^{2} \leftarrow \cdots \leftarrow R_{i}^{2 L} \stackrel{\tau_{2 k}}{\longrightarrow} S_{i}\right) .
$$

Obviously, such a zig-zag exists and $L$ can be chosen independently on $i$. Then the following square commutes for each odd $j$ :

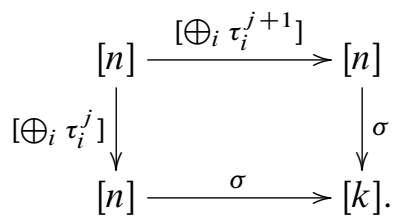

Hence, the application of the second equivariance condition of definition $6.1 \mathrm{~L}$ times gives the second Fiedorowicz equivariance condition.

For the first equivariance condition we do an analogous construction by choosing a presentation of the braid $\rho$ as an image of a zigzag.

Let $A$ be an operad in the sense of 2.3. We construct an operad $B$ in the sense of 6.1 as follows. As a braided collection $B$ coincides with $A$. Its multiplication is the same as in $A$ as well. The only nontrivial statement to check is that $B$ satisfies the equivariance conditions from Definition 6.1. To prove the second condition we use Lemma 5.2.

It is obvious also that the first equivariance condition is satisfied in the following special case. Let $\sigma^{\prime}: T \rightarrow S^{\prime}$ be an order-preserving map and let $\rho: S^{\prime} \rightarrow S$ be a quasibijection. Apply Lemma 3.1 to produce a quasibijection $\pi\left(\rho, \sigma^{\prime}\right): T^{\prime} \rightarrow T$ and order-preserving map $\sigma\left(\rho, \sigma^{\prime}\right): T \rightarrow S$ such that $\sigma^{\prime} \cdot \rho=\pi\left(\rho, \sigma^{\prime}\right) \cdot \sigma\left(\rho, \sigma^{\prime}\right)$. Then $b\left(\pi\left(\rho, \sigma^{\prime}\right)\right)=\Gamma_{B}(b(\rho) ; 1, \ldots, 1)$ and we can apply the first equivariance Fiedorowicz condition.

Then the first equivariance condition is satisfied in general because of the second equivariance condition of the Definition 6.1 applied to the commutative diagram

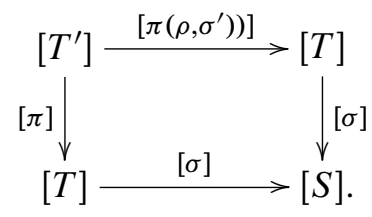


Theorem 6.2. The category of $Q_{n}$-operads $3 \leq n \leq \infty$ and the category of symmetric operads are equivalent.

Proof. The proof is a repetition of the above proof with the simplification that $s_{n}: \Pi Q_{n} \rightarrow \delta$ for $3 \leq n \leq \infty$ is an equivalence.

\section{Locally constant $n$-operads}

The quasisymmetric $n$-operads are defined in any symmetric monoidal category $V$. But according to Theorems 6.1 and 6.2 they are different from symmetric operads only when $n=1,2$. As we have seen before the main reason why quasisymmetric operads collapse to symmetric operads for $n>2$ is that the configuration space $\operatorname{Conf}_{k}\left(\mathbb{R}^{n}\right)$ is simply connected and so localising with respect to quasi-bijections can only produce a groupoid equivalent to $\delta$. The correct procedure, therefore, should be to take the weak $\omega$-groupoid $\Pi_{\infty} Q_{n}$ and consider presheaves on it with values in $V$ as the category of collections. There are, however, considerable technical difficulties with this approach.

Fortunately, the results of Cisinski [5] show a way around this problem by considering as the category of collections the category of locally constant functors from $Q_{n}^{\text {op }}$ to $V$. Pursuing this idea we give the following definition.

Definition 7.1. Let $V$ be a symmetric monoidal category and $\mathcal{W}$ (weak equivalences) be a subclass of its morphisms. A locally constant $n$-operad in $(V, \mathcal{W})$ is an $n$-operad $A$ in $V$ such that for every quasibijection $\sigma: T \rightarrow S$ the morphism $A(\sigma): A_{S} \rightarrow A_{T}$ is a weak equivalence.

Remark. We have chosen the name locally constant $n$-operads (which some people prefer to call homotopically locally constant $n$-operads) for two reasons. First, we would like our terminology to agree with the terminology of [5]. But a more important reason is about philosophy. The notion of locally constant $n$-operad (and locally constant functor) depends only on the class of weak equivalences but not on the choice of homotopy theory in $V$. For example, if $V$ is a symmetric monoidal category and Iso is the class of all isomorphisms, a locally constant $n$-operad in ( $V$, Iso) is the same as a quasisymmetric $n$-operad in $V$. So, the word 'homotopical' is a little bit misleading. Compare this situation with the theory of homotopy limits developed in [6]. We believe that a 'true' reason for this phenomenon is that homotopy limit and locally constant functors are higher categorical rather than homotopical notions. But the homotopy theory is helpful in computations. As far as we know a similar argument is behind Cisinski's choice of terminology.

An example of an interesting locally constant $n$-operad in the model category of topological spaces which is not a quasisymmetric $n$-operad is the Getzler-Jones 
$n$-operad $\mathbf{G} \mathbf{J}^{n}$ constructed in [1] for all $n<\infty$. One can also construct an $\infty$-version $\mathbf{G} \mathbf{J}^{\infty}$ by the formula $\mathbf{G} \mathbf{J}_{T}^{\infty}=\mathbf{G} \mathbf{J}_{\bar{T}}^{n}$, where $<_{-n}$ is the minimal nonempty relation in the $\infty$-ordinal $T$, the $n$-ordinal $\bar{T}$ has the same underlying set as $T$ and the relation $<_{n-p-1}$ in $\bar{T}$ coincides with the relation $<_{-p}$ in $T$.

Let $V$ be a symmetric monoidal category equipped with a class of weak equivalences $\mathcal{W}$. We introduce the following notations:

- $\mathrm{SO}$ is the category of symmetric operads in $V$;

- $\mathrm{BO}$ is the category of braided operads in $V$;

- $\mathrm{O}_{n}$ is the category of $n$-operads in $V$;

- $\mathrm{QO}_{n}$ is the full subcategory of $\mathrm{O}_{n}$ of quasisymmetric $n$-operads in $V$;

- $\mathrm{LCO}_{n}$ is the full subcategory of $\mathrm{O}_{n}$ of locally constant $n$-operads in $(V, \mathcal{W})$.

Definition 7.2. A morphism of operads (in any of the categories above) is a weak equivalence if it is a termwise weak equivalence of the collections. The homotopy category of operads is the category of operads localised with respect to the class of weak equivalences.

Let us describe the relations between the different categories of operads we deal with in this paper. We have already done it for the case $\mathcal{W}=$ Iso in Section 6.

Let us fix a base symmetric monoidal model category $V$ and let $\mathcal{W}$ be its class of weak equivalences in the model category theoretic sense. Moreover, we will assume that $V$ satisfies the conditions from Section 5 of [1], which means that there is a model structure on the category of collections transferable to the category of operads (see [1] for the details).

For $n=1$ the relationships between operadic categories above is simple. The following categories are isomorphic to the category of nonsymmetric operads

$$
\mathrm{O}_{1} \simeq \mathrm{LCO}_{1} \simeq \mathrm{QO}_{1}
$$

and we have a classical adjunction between nonsymmetric operads and symmetric operads. All this is true on the level of homotopy categories.

For $n=2$ we have the following diagram of categories and right and left adjoint functors:

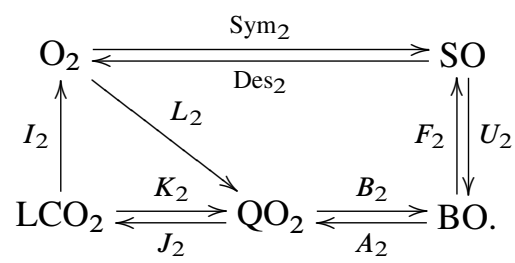

In this diagram the functor $\mathrm{Des}_{2}$ is right adjoint to $\mathrm{Sym}_{2}$ (see [1], [2] for the construction). The functors $I_{2}$ and $J_{2}$ are natural inclusions. The functor $K_{2}$ is left adjoint to $J_{2}$ and $L_{2}$ is left adjoint to the composite $J_{2} \cdot I_{2}$. Using the theory of 
internal operads from [2] one can show that $L_{2}$ on the level of collections is given by the left Kan extension along the localisation functor $l_{2}: Q_{2} \rightarrow \Pi Q_{2}$ :

$$
L_{2}(A)=\operatorname{Lan}_{l_{2}}(A) .
$$

We have also the same formula for $K_{2}$. The functor $A_{2}$ is a right adjoint and $B_{2}$ is a left adjoint part of the equivalence constructed in Section 6. Finally, $U_{2}$ is the functor which produces a braided operad from a symmetric operad by pulling back along the functor $p: \mathscr{B} r \rightarrow S$ and $F_{2}$ is its left adjoint given by quotienting with respect to the action of the pure braid groups .

Theorem 7.1. The homotopy category of locally constant 2-operads and the homotopy category of quasisymmetric 2-operads and the homotopy category of braided operads are equivalent.

The functor of symmetrisation $\mathrm{Sym}_{2}$ can be factorised as $L_{2} \cdot B_{2} \cdot F_{2}$.

A base space $X$ is a 2-fold loop space (up to group completion) if and only if it is an algebra of a contractible 2-operad, if and only if it is an algebra of a contractible braided operad (Fiedorowicz's recognition principle [4]).

Proof. Since $\Pi Q_{2}$ is a groupoid, the localisation functor $l_{2}$ is locally constant in the sense of [5], 1.14. By the formal Serre spectral sequence [5], Prop. 1.15, we get that the homotopy left Kan extension along $l_{2}$ is a left adjoint to the restriction functor between homotopy categories of collections. The functor $l_{2}$ induces a weak equivalence of the nerves and so, by Quillen's Theorem B, it is also aspherical in the sense of [5], 1.4. So, by [5], Prop. 1.16, the homotopy left Kan extension along $l_{2}$ is an equivalence of homotopy categories of collections.

Taking into account the formula (4) we see that to prove the equivalence of homotopy categories of operads it is enough to show that for an $n$-operad $A(1 \leq n \leq \infty)$ there exists a cofibrant replacement $B(A)$ such that the underlying $Q_{n}$-collection of $B(A)$ is cofibrant in the projective model structure.

Recall [1] that $\mathbf{p h}^{n}$ is the categorical symmetric operad representing the 2-functor of internal pruned $n$-operads. In particular an $n$-operad $A$ is represented by an operadic functor $\tilde{A}: \mathbf{p h}^{n} \rightarrow V^{\bullet}$. If we forget about operadic structures then for any $k \geq 0$ we will have a functor $\tilde{A}_{k}: \mathbf{p h}_{k}^{n} \rightarrow V$. Take the bar-resolution $B(L, L, C(\tilde{A}))$, where $(L, \mu, \epsilon)$ is the monad on the functor category $\left[d\left(\mathbf{p h}^{n}\right), V\right]$ generated by restriction and left Kan extension along the inclusion of discretisation $d\left(\mathbf{p} \mathbf{h}_{k}^{n}\right)$ of $\mathbf{p h}{ }_{k}^{n}$ to $\mathbf{p h}{ }_{k}^{n}$ and $C(A)$ is the termwise cofibrant replacement of the underlying $n$-collection of $A$. These functors for all $k \geq 0$ form an operadic functor $\mathbf{B}(A): \mathbf{p h}^{n} \rightarrow V^{\bullet}$ and, hence, determine an $n$-operad $B(A)$ which is a cofibrant replacement for $A$ [2].

Since $\mathbf{B}_{k}(A)$ is a bar-construction on cofibrant collection it is cofibrant in the projective model category of functors. Recall also that there is a symmetric categorical operad $\mathbf{r h}^{n}$ representing the 2-functor of internal reduced $n$-operads [1] and a projection $p: \mathbf{p h}^{n} \rightarrow \mathbf{r h}^{n}$. A typical fiber (in a strict sense) of this projection over an object $w \in \mathbf{r h}^{n}$ is a category with a terminal object $s(w)$. The map $s$ assembles to 
the (nonoperadic) functor $s: \mathbf{r h}^{n} \rightarrow \mathbf{p h}^{n}$, which is by definition a section of $p$ and is also a right adjoint to $p$. The counit of this adjunction is the identity and the unit is the unique map to the terminal object $s(w)$.

The simple calculations with this adjunction show that the restriction functor $s^{*}$ preserves the cofibrant objects for projective model structures and so $s^{*}\left(\mathbf{B}_{k}(A)\right)$ is cofibrant.

There is also an inclusion $j: J_{n}^{\mathrm{op}} \rightarrow \mathbf{r h}^{n}$ [1]. It is not hard to see also that the categories $J_{n}^{\mathrm{op}}(k)$ and $\mathbf{r h}_{k}^{n}$ are Reedy categories. Recall that the objects of $\mathbf{r h}_{k}^{n}$ are planar trees decorated by pruned $n$-trees (i.e., $n$-ordinals). One can choose the total number of edges of $n$-trees in a decorated planar tree as a degree function and see that each morphism decreases strictly this function. ${ }^{1}$

It follows from these considerations that the functor $s^{*}\left(\mathbf{B}_{k}(A)\right)$ satisfies the following property characterising cofibrant objects in the projective model categories for functor categories over Reedy categories:

$$
\operatorname{colim}\left(s^{*}\left(\mathbf{B}_{k}(A)\right)(w)\right) \rightarrow s^{*}\left(\mathbf{B}_{k}(A)\right)(T)
$$

is a cofibration. Here the colimit is taken over the category of all $w \rightarrow T, w \neq T$ in $\mathbf{r h}_{k}^{n}$. It was proved in [1] that $J_{n}^{\mathrm{op}}(k)$ is cofinal in $\mathbf{r h}_{k}^{n}$. Exactly the same argument shows that in the colimit (5) one can replace $w \in r h_{k}^{n}$ with the objects from $J_{n}^{\mathrm{op}}(k)$. And, therefore the restriction $j^{*} s^{*}\left(\mathbf{B}_{k}(A)\right)$ is cofibrant as well.

The quotient functor $q: J_{n}^{\mathrm{op}}(k) \rightarrow Q_{n}^{\mathrm{op}}(k)$ induces the restriction functor $q^{*}$ on functor categories which is fully faithful. It follows from this that $q^{*}$ reflects cofibrations. We observe that

$$
q^{*}\left(u(B(A))=j^{*} s^{*}\left(\mathbf{B}_{k}(A)\right),\right.
$$

and so $u(B(A))$ is cofibrant. Hence the first statement of the theorem is proved.

The statement about symmetrisation is obvious since $\operatorname{Des}_{2}=U_{2} \cdot A_{2} \cdot J_{2} \cdot I_{2}$. Finally, a contractible operad is locally constant, so the third statement follows from the first statement, Theorem 8.6 from [1] and the fact that the functors $U_{2}, A_{2}, J_{2}, I_{2}$ preserve endomorphism operads.

For $3 \leq n \leq \infty$ the corresponding diagram is

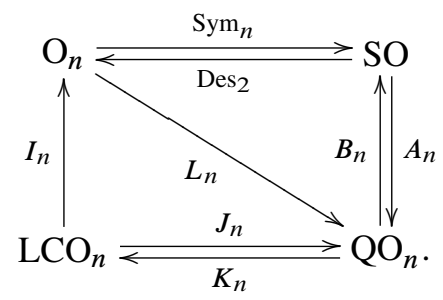

${ }^{1}$ In fact, $\mathbf{r h}_{k}^{n}$ is a poset, but we did not provide a proof of this fact in [1]. 
Theorem 7.2. For $3 \leq n \leq \infty$ the category of symmetric operads is equivalent to the category of quasisymmetric $n$-operads.

For $3 \leq n<\infty$ a base space $X$ is an $n$-fold loop space (up to group completion) if and only if it is an algebra of a contractible n-operad.

The homotopy category of locally constant $\infty$-operads, the homotopy category of quasisymmetric $\infty$-operads and the homotopy category of symmetric operads are equivalent.

A base space $X$ is an infinite loop space (up to group completion) if and only if it is an algebra of a contractible $\infty$-operad if and only if it is an algebra of a contractible symmetric operad (May's recognition principle [7]).

Proof. The proof is analogous to the proof of Theorem 7.1.

An interesting question which we do not consider here is the existence of model structures on the various categories of operads. The results of [5] indicate that this might be possible. But it is a subject for a future paper.

Acknowledgements. I would like to thank Denis-Charles Cisinski for his nice answers [5] to my sometimes naive questions. I wish to express my gratitude to C. Berger, I. Galvez, E. Getzler, V. Gorbunov, A. Davydov, R. Street, A. Tonks, M. Weber for many useful discussions and to the anonymous referee for useful comments concerning the presentation of the paper.

I also gratefully acknowledge the financial support of Scott Russel Johnson Memorial Foundation, Max Plank Institut für Mathematik and Australian Research Council (grant No. DP0558372).

\section{References}

[1] M. A. Batanin, Symmetrisation of $n$-operads and compactification of real configuration spaces. Adv. Math. 211 (2007), 684-725. Zbl 1146.18003 MR 2323542 243, 245, 250, $251,259,260,261$

[2] M. A. Batanin, The Eckmann-Hilton argument and higher operads. Adv. Math. 217 (2008), 334-385. Zbl 1138.18003 MR 2365200 240, 245, 247, 259, 260

[3] C. Berger, Combinatorial models for real configuration spaces and $E_{n}$-operads. In Operads: Proceedings of Renaissance Conferences (Hartford, CT/Luminy, 1995), Contemp. Math. 202, Amer. Math. Soc., Providence, RI, 1997, 37-52. Zbl 0860.18001 MR 1436916 250

[4] Z. Fiedorowicz, The symmetric bar construction. Preprint 1992. http://www.math.ohio-state.edu/ fiedorow/ 241, 260

[5] D.-D. Cisinski, Locally constant functors. Math. Proc. Cambridge Philos. Soc. 147 (2009), 593-614. Zbl 05635886 MR 2557145 258, 260, 262

[6] W. G. Dwyer, P. S. Hirschhorn, D. M. Kan, and J. H. Smith, Homotopy limit functors on model categories and homotopical categories. Math. Surveys Monogr. 113, Amer. Math. Soc., Providence, RI, 2004. Zbl 1072.18012 MR 2102294258 
[7] J. P. May, The geometry of iterated loop spaces. Lectures Notes in Math. 271, SpringerVerlag, Berlin 1972. Zbl 0244.55009 MR 0420610 238, 262

Received May 29, 2008; revised January 17, 2009

M. A. Batanin, Department of Mathematics, Macquarie University, NSW 2109, Australia

E-mail: mbatanin@ics.mq.edu.au 